private homes without prior permission from the district attorney. Involuntary electroconvulsive therapy (ECT) without second opinion for up to three initial sessions became legitimate. The role of patients' rights committees was diminished.

Violations of the human rights of people who are mentally ill are recognised globally (Drew et al, 2011). The Egyptian experience underlines the importance of public education and community participation in the drafting of new legislation. Ideally, laws are ratified to fulfil society's need for regulation. Bringing in new ideas of human rights and empowerment of service users becomes a challenge when the predominant culture does not fully acknowledge the rights of people with a mental illness.

Nevertheless, the process of drafting the Act with repeated conferences and workshops, lengthy parliamentary debates in the upper and lower houses, and substantial media coverage - has had an effect on the wider community's perception of people who are mentally ill, their place in society and their role. Patients are their own best advocates. Bringing a long-stay patient to meet with MPs in the Egyptian parliament was probably the loudest call that led to Egypt's Mental Health Act 2009.

\section{References}

Drew, N., Funk, M., Tang, S., et al (2011) Human rights violations of people with mental and psychosocial disabilities: an unresolved global crisis. Lancet, 378, 1664-1675.

Government Press Cairo (1922) Annual Report 1921, Lunacy Division, Egyptian Ministry of the Interior. Government Publications Office, Ministry of Finance.

Tuke, W. S. \& Urquhart, A. R. (1879) Two visits to the Cairo Asylum, 1877 and 1878. Journal of Mental Science, 25, 43-48.

Zaki, M. M. (2009) Egyptian legislation on the compulsory treatment of persons with psychiatric disorders: the old and the new. International Psychiatry, 6, 57-58.

\section{RESEARCH} PAPER

\title{
The 'revolving door': a profile of acute admissions at a South African psychiatric hospital
}

\author{
Zahir Vally ${ }^{1,2}$ and Nasera Cader ${ }^{1,2}$
}

'Lentegeur Psychiatric Hospital, Cape Town, South Africa, email z.vally@pgr.reading.ac.uk

${ }^{2}$ Department of Psychiatry and Mental Health, University of Cape Town, South Africa

The authors wish to thank Felicity Haigh for her assistance in completing the data collection.
This paper reports admission rates within the acute service at a major South African tertiary psychiatric facility, Lentegeur Psychiatric Hospital (LPH) in Cape Town.

The acute service in South African psychiatric hospitals is a challenging environment in which clinicians are faced with a multitude of factors that impinge on the efficacy of their interventions. This environment is characterised by lengthy and constantly growing waiting lists, an ever-present pressure to vacate hospital beds for incoming patients and a noticeable increase in the clinical presentation of severe Axis I diagnoses (Strebel $e t$ $a l, 1999)$. Moreover, the social context from which these patients emerge compounds the burden on services and should be considered in planning treatment. There is widespread poverty, the vast majority reside in informal housing on the outskirts of the urban cities, there is mass unemployment, some of the highest rates of substance misuse in the world, and an increasing prevalence of HIV and other communicable diseases. This is an important consideration, given that there is emerging evidence from low- and middle-income countries that mental ill-health is strongly associated with poverty and aspects of social deprivation
(Patel \& Kleinman, 2003). These external psychosocial factors in turn contribute to poor discharge planning, the premature discharge of patients who clinically would require a lengthier hospital stay and, ultimately, speedy readmission following discharge. This phenomenon is most often captured by the term 'revolving door', coined to describe patients who are admitted at least three times during their lifetime (Webb et al, 2007). Findings in the UK suggest that readmission rates among patients with severe psychiatric disorders are high: approximately $50 \%$ of patients admitted have had previous psychiatric admissions, and about $40 \%$ of patients require rehospitalisation within 1 year of discharge (Sweetman \& Davies, 2004).

There are a number of factors that appear to predict readmission. The majority of studies suggest that the number of previous psychiatric admissions is a good predictor of the risk of readmission (e.g. Webb et al, 2007; Bowersox, 2009). This is often the most reliable indicator. Of those readmitted, between one-half and two-thirds are readmitted within 12 months of discharge, suggesting that this is a high-risk period. The most critical point is 1 month after discharge. Higher rates of readmission are associated with a diagnosis of schizophrenia and other psychosis. A diagnosis of substance misuse or dependence is also associated 
Table 1

Sample characteristics $(n=666)$

\begin{tabular}{|l|c|}
\hline Variables & Frequency (\%) \\
\hline Age (years) & \\
\hline $18-24$ & $122(18.2)$ \\
\hline $25-34$ & $206(30.9)$ \\
\hline $35-49$ & $238(35.7)$ \\
\hline $50-59$ & $85(12.7)$ \\
\hline $60+$ & $15(2.2)$ \\
\hline & \\
\hline Differential diagnosis & \\
\hline Schizophrenia spectrum & $177(26.5)$ \\
\hline Affective spectrum & $246(36.9)$ \\
\hline Substance-induced spectrum & $27(4.1)$ \\
\hline Brief-episode spectrum & $150(22.5)$ \\
\hline Secondary to a general medical condition & $63(9.5)$ \\
\hline Other & $3(0.5)$ \\
\hline & \\
\hline Catchment area & \\
\hline Urban & $552(82.7)$ \\
\hline Rural & $86(12.9)$ \\
\hline Not part of catchment area & $28(4.2)$ \\
\hline & \\
\hline Type of admission & \\
\hline First & $359(53.9)$ \\
\hline Repeat & $307(46.1)$ \\
\hline & \\
\hline
\end{tabular}

with more frequent admissions, as are chronic psychiatric disorders with an affective component. Sweetman \& Davies (2004) found that patients with a dual diagnosis have twice as many admissions per year as those with a single diagnosis. Furthermore, research indicates that individuals who are compliant with their psychotropic medication have a lower rate of readmission. Studies have indicated that more detailed planning at discharge can be a protective factor against future readmission. Age also appears to be a factor that affects readmission, with younger individuals being more likely to be readmitted early (Fontanella, 2008). Nonetheless, McGurk \& Mueser (2008) found that older patients with severe mental illness were more cognitively impaired and thus less responsive to cognitively based interventions. This is a factor which could result in readmission.

\section{Method}

The present study is archival and reports retrospective data from March 2009 to February 2011 for all 666 patients admitted to the acute service at Lentegeur Psychiatric Hospital (LPH) during this period. All patients are examined by a medical officer and assessed using the Maudsley psychiatric interview; a provisional diagnosis is assigned upon admission. The following patient characteristics were recorded: age, household location and provisional diagnosis. Then the number of admissions for each patient within the 2-year time frame was counted and the periods between each episode calculated.

\section{Results and discussion}

The 'revolving door' refers to the expectation that patients will present frequently for readmission over a short period of time. However, in stark
Table 2

Readmission data $(n=666)$

\begin{tabular}{|l|c|}
\hline & Frequency (\%) \\
\hline Number of admissions & \\
\hline 1 & $359(53.9)$ \\
\hline 2 & $111(16.7)$ \\
\hline 3 & $36(5.4)$ \\
\hline 4 & $11(1.7)$ \\
\hline 5 & $7(1.1)$ \\
\hline Previous admission prior to March 2009 & $142(21.3)$ \\
\hline & \\
\hline One relapse & \\
\hline Within 1 week & 1 \\
\hline Within 1 month & 7 \\
\hline Within 6 months & 16 \\
\hline Within 1 year & 21 \\
\hline More than 1 year & 19 \\
\hline & \\
\hline Two relapses & \\
\hline Within 1 week & 0 \\
\hline Within 1 month & 0 \\
\hline Within 6 months & 10 \\
\hline Within 1 year & 3 \\
\hline More than 1 year & 1 \\
\hline & \\
\hline Three relapses & 0 \\
\hline Within 1 week & 0 \\
\hline Within 1 month & 2 \\
\hline Within 6 months & 0 \\
\hline Within 1 year & 0 \\
\hline More than 1 year & \\
\hline & 0 \\
\hline Four relapses & \\
\hline Within 1 week & \\
\hline Within 1 month & \\
\hline Within 6 months & \\
\hline Within 1 year & \\
\hline More than 1 year & \\
\hline & \\
\hline & \\
\hline & \\
\hline & \\
\hline & \\
\hline
\end{tabular}

contradiction to this expectation, the current retrospective study reveals a lower than anticipated readmission rate, with similar rates of first admissions (54\%) and readmissions (46\%) (Tables 1 and 2). Furthermore, when patients were readmitted for a second (or later) episode, this was often after a period exceeding 2 years. It seems that a large proportion of patients who do indeed relapse do so long after discharge, certainly well after 2 years (beyond the period examined here).

Patients presented with a minimum of one (16.7\%) to a maximum of five relapse episodes (1.1\%). Most patients who presented for readmission did so only once and within 1 year of discharge. A similar trend is evident for the other groups who presented more often. This is an interesting finding given that the literature strongly suggests that frequent admissions over a short period of time should be expected, certainly in Western samples (Sweetman \& Davies, 2004). Why might this be different in South Africa?

- The ethos common to the indigenous African culture - encompassing a sense of community, and social, decentralised care by families and larger community structures - may reduce the burden on formal mental health services. It also assists patient compliance with post-discharge treatment. 
- The Health Professionals Council of South Africa has introduced a compulsory system of continuing professional development to encourage mental health staff to improve their clinical knowledge and skills, especially with regard to ethical, evidence-based services. Education and implementation of powerful evidence-based interventions whose efficacy is empirically validated ensure that patients receive the best management possible, potentially contributing to favourable patient outcomes.

- The inception of the Mental Health Care Act 2000 and a recent move towards deinstitutionalisation have had an impact on the pathways of care and consequently the nature of presentations to tertiary-level psychiatric facilities such as LPH. Patients in the catchment area of this hospital now have access to psychiatric care (usually by a registrar) and are subject to a compulsory 72-hour observation period at a district-level facility. It is only when patients become unmanageable or symptoms persist for longer than 72 hours that they are transferred to a tertiary facility. As a result, the smaller than expected readmission rate observed here may suggest that a larger than anticipated proportion of patients remit during those first few days and do not require further admission to acute care. Moreover, the low incidence of substance-induced disorders (SIDs) - at $4 \%$ - in this sample is contrary to that expected, given the widespread prevalence of substance misuse in this catchment area. It is plausible that most SIDs dissipate within a short period of time (while patients are at district facilities) and therefore most may never require tertiary care.

- Despite the commonly held belief that South Africa suffers from an underresourced public health service and clinicians feel unable to render optimal care due to the pressure of contextual challenges, it seems that adequate treatment is nevertheless being provided at this particular hospital and patients seem to derive a sense of benefit from the interventions received, potentially resulting in longer periods of remission.

Our results concur with previous findings regarding the high prevalence of affective disorders in psychiatric samples (36.9\%; Loveland-Cook et al, 2010), the tendency for younger patients to present with more severe symptoms and a greater likelihood of readmittance (22.9\%; Fontanella, 2008) and the relatively high incidence of readmissions in the older age category, where it has been suggested that patients may be unable to adequately utilise the psychotherapeutic services offered to them (McGurk \& Mueser, 2008).
The data indicate that the periods between episodes increase progressively over time, suggesting that patients remain well for longer periods over the course of their illness. We cannot with any certainty conclude why this may be so. However, it may be the result of the natural psychological process of coming to terms with the psychiatric diagnosis, accompanied by acceptance of the prescribed treatment. Furthermore, having received comprehensive multidisciplinary input, including evidence-based interventions over an extended period of time, as well the fact that patients may be more amenable to accepting and acting upon the input received, may result in extended positive outcomes. Researchers studying reasons for attrition have found that there were few differences for patients who remain compliant and those who drop out. One difference was that patients who were satisfied with their level of treatment did not drop out of the study (Primm et al, 2000).

It is important to bear in mind that these results pertain to one hospital in a particular catchment area situated reasonably close to a major city centre. This may not reflect what happens in hospitals elsewhere in South Africa, which may not be as well resourced. Patients at LPH are able to access the services of a complete multidisciplinary team and a bio-psychosocial approach to treatment which includes psychiatric and medical input, psychological services (e.g. psychotherapy groups, individual psychotherapy, family psychoeducation), occupational therapy, physiotherapy, nutrition/dietetics and social work, as part of a complete package of care.

\section{References}

Bowersox, N. W. (2009) Treatment Attrition and Relapse Readmission in Psychiatric Inpatients: Predictors of Treatment Engagement and Psychiatric Relapse. Dissertation, Marquette University.

Fontanella, C. A. (2008) The influence of clinical, treatment and healthcare system characteristics on psychiatric readmission of adolescents. American Journal of Orthopsychiatry, 78, 187-198.

Loveland-Cook, C. A., Flick, L. H., Homan, S. M., et al (2010)

Psychiatric disorders and treatment in low income pregnant women. Journal of Women's Health, 19, 1251-1262.

McGurk, S. R. \& Mueser, K. T. (2008) Response to cognitive rehabilitation in older versus younger persons with severe mental illness. American Journal of Psychiatric Rehabilitation, 11, 90-105.

Patel, V. \& Kleinman, A. (2003) Poverty and common mental disorders in developing countries. Bulletin of the World Health Organization, 81, 609-615.

Primm, A. B., Gomez, M. B., Tzolova-Tontchev, I., et al (2000) Severely mentally ill patients with and without substance abuse disorders: characteristics associated with treatment attrition. Community Mental Health Journal, 36, 235-246.

Strebel, A., Stacey, M. \& Msomi, N. (1999) Gender and psychiatric diagnosis: a profile of admissions to mental hospitals in the Western Cape Province, South Africa. Archives of Women's Mental Health, 2. 75-81.

Sweetman, C. \& Davies, S. (2004) Factors involved in psychiatric hospital readmissions: a review of current literature. Current Medical Literature (Psychiatry), 15, 61-66.

Webb, S., Yaguez, L. \& Langdon, P. E. (2007) Factors associated with multiple readmissions to a psychiatric hospital. Journal of Mental Health, 16, 647-661. 